\title{
Development of PINN Controller for Fuel Handling System of Pressurized Heavy Water Reactors
}

\author{
Dae Yeong LIM", ${ }^{1,2}$ Sehi PARK ${ }^{1}$, Kil To CHONG ${ }^{1,2^{*}}$ \\ ${ }^{1}$ Dept. of Electronics \& Information Engineering, Chonbuk National University, Jeonju, 54896, Korea \\ tamudylim@gmail.com,sehibark@gmail.com \\ ${ }^{2}$ Advanced Electronic \& Information Research Center, Chonbuk National University, Jeonju, 54896, Korea \\ kitchong@jbnu.ac.kr(*Correspondingauthor)
}

\begin{abstract}
The required operating pressure of the Wolsong nuclear power plant is currently controlled by a proportional integral (PI) controller. The PI controller has a simple structure and was designed to meet requirements through gain setting. However, these control requirements can be difficult to meet without properly adjusting the gain when certain parameters change, such as the wear and tear in the valves or pipes. To solve these problems, it is important to dynamically change the PI controller gain or compensate for the PI controller output. The purpose of this study is to help design a controller that is capable of providing stable control in order to reduce errors regardless of parameter changes. The proposed PI neural network (PINN) control technique involves a PI controller and a neural network controller combined in parallel. The neural network component which is designed to be robust compensates the output of the controller for changes in the above-mentioned parameters. Because assessing the controller performance straightforwardly in real-time processes can be difficult, a simulator model was developed based on real-time processes, and it showed changes in the parameters involved. The results confirmed that the proposed PINN controller reduced the instability of the fuel supply machine and, hence the aforementioned problem could be properly controlled.
\end{abstract}

Keywords: Heavy water reactors, Pressure control, Neural network control, Power plant.

\section{Introduction}

Nuclear power plants are categorized based on the different types of reactors they employ and there are five types of reactors widely used in the world, namely: Pressurized water reactors (PWRs), Boiling water reactor(BWRs), Heavy-water reactors(HWRs), Gas cooled reactor(GCRs), and Liquid Metal-cooled Fast Breeder Reactor(LMFBRs) (Kadiyala, Kommalapati \& Huque, 2016). These reactors use different coolant combinations as well as different uranium and plutonium combinations to generate power. PWRs and BWRs use light water to cool down their cores whereas HWRs like the CANada Deuterium Uranium(CANDU) reactor utilize heavy water. This paper analyses the Fuel Handling System for a pressurized heavy water reactor similar to CANDU (Griffiths, 2014).

The replacement system of the nuclear reactor is denoted as Fuel Handling $(\mathrm{F} / \mathrm{H})$ (Katoh et al., 2010; Zeng et al., 2018), which is the control system of the nuclear replacement robot used to supply pressure, and the nuclear replacement robot is the fuelling machine $(\mathrm{F} / \mathrm{M})$ (Shah, Hong \& Choi, 2017; Banerjee \& Gupta, 2017). The F/H system is located in front of the $\mathrm{F} / \mathrm{M}$, and controls the supply pressure of the heavy water required to exchange nuclear fuel. The drive pressure control system of $\mathrm{F} / \mathrm{H}$ which is discussed in this paper operates to provide efficient pressure within a range that does not exceed the safety setpoint depending on the set mode. The control system for the driving pressure of the $\mathrm{F} / \mathrm{H}$ is currently operated by a PI controller and is presented in section 4.1. The PI control method is used in many types of power plants, such as nuclear (Zarei, 2018; Lamba, Singla \& Sondhi, 2017), solar (Beschi, Padula \& Visioli, 2016; Rahman, Saikia \& Sinha, 2016), and thermal power plants (Zeng et al., 2019) because it involves a simple structure which is easy to implement and provides stable control.

However, it is difficult for the PI control method to achieve the required stability and steady state since the system has nonlinear dynamic characteristics due to parameter change or disturbance. Therefore, in order to overcome the limits of the PI control used in the abovementioned process, the following control methods have been proposed.

When the time delay is unknown or can be changed and the system degree is not exactly known, the Long Range Predictive Control (LRPC) method was applied (Prasad, Swidenbank \& Hogg, 1998). For system optimization, in terms of the nonlinearity of the system and the nonminimum phase characteristics, Model Predictive control (MPC) (Vazquez et al., 2017; Liu, Zhang \& Lee, 2016; Zhang \& Shen, 2016) was explored. To overcome disturbance due to unexpected 
changes, fuzzy PID control based on fuzzy gain Scheduling method (Qin et al., 2018; Nikolovski, Reza Baghaee \& Mlakić, 2018) was proposed.

To develop the controller using fuzzy control technique, the knowledge of skilled professionals is required and the MPC controller requires a relatively similar transfer function. When the parameter estimation applied is inadequate, LRPC can be greatly affected by system disturbances because accurate controller design is difficult. On the other hand, a great deal of research has been carried out on artificial intelligence control based on the input/output data of the uncertainty model (Li, Wang \& Goel, 2015; Mahajan et al., 2018). A typical example of artificial intelligence control is neural networks (NN) wherein neural network algorithms can improve control performance without direct modeling. In (Liu et al., 2015; Heravi \& Eslamdoost, 2015; Borkowski, 2017), these types of algorithm were applied to various power plants. Recently, a hybrid control method exploiting the advantages of $\mathrm{NN}$ has been studied (Attaran, Yusof \& Selamat, 2016; Azali \& Sheikhan, 2016; Sharma et al., 2016). This method can adapt when system parameter changes or disturbance occurs.

Therefore, in this paper, an NN controller is connected in parallel to the conventional PI control method in order to control the $\mathrm{F} / \mathrm{H}$ system. Because of the PI controller's simple structure and high reliability, it was utilized as the main controller to compensate the linear components. The NN controller was used as an additional controller to compensate for changing parameters and disturbances. The system was modelled using Simulink of Matlab in order to verify its performance. The real system characteristics were mathematically represented during simulations with parameters for valve, volume and pressure. The valve characteristics curve and slew rate parameter values of the $\mathrm{F} / \mathrm{H}$ system were adjusted in order to validate the performance of the proposed controller. Simulation results showed that the proposed PINN controller was more stable compared to PI controller when the parameters were changed as well as in the presence of disturbances.

This paper is structured as follows. The driving pressure control system of the $\mathrm{F} / \mathrm{H}$ is described in Section 2. The simulated system is discussed in Section 3 followed by the presentation and simulation of the proposed PINN controller in Sections 4 and Section 5, respectively. Finally, the concluding remarks are given in section 6 .

\section{Fuel Handling System}

The purpose of the $\mathrm{F} / \mathrm{H}$ is to replace the eight fuel bundles twice a day. While refueling, the operation pressure is set depending on the behavior of the processor. This paper focuses on the pressure control system for the replacement of fuel bundles which is denoted as the $\mathrm{F} / \mathrm{H}$ system. The drive pressure is set as the operation mode depending on the processing behavior. The role of the operation mode is to minimize the influence exerted to $\mathrm{F} / \mathrm{H}$ by the sudden load change within the process system and to stabilize the pressure within the F/H system. In this section, the detailed components are introduced and the role of each system is described. The drive pressure of the $\mathrm{F} / \mathrm{H}$ is divided into the process and the control systems.

\subsection{Process System}

The block diagram of $\mathrm{F} / \mathrm{H}$ is presented in figure 1. It consists of two pumps, only one of which is operated in steady state. The Common Bleed Valve is indicated by the term COMMON in the block in Figure 1 and consists of a valve ( $P C V 11)$, a pressure instrument $(P T 11)$, and a controller $(P C 11)$. The $\mathrm{A}$-side and $\mathrm{C}$-side have the same mechanism, and refueling is operated according to the pressure between A-side and C-side. The A-SIDE block consists of two valves, a measuring instrument, and a controller for providing the driving pressure of the $\mathrm{F} / \mathrm{M}$. The two valves are the Series Valve $(P C V 11 \# 1(A))$ and the shunt valve $(P C V 11 \# 2(A))$. The role of the aforementioned instrument is to measure the pressure between the two valves using the pressure sensor $P T 11(A)$, while $P C 11(A)$ is controlled by each opening valve.

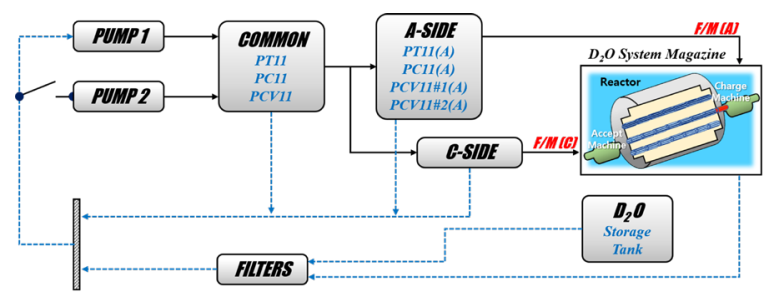

Figure 1. F/H system block diagram

Examination of the heavy water in the circulation process reveals that the volume of the residual $\mathrm{D}_{2} \mathrm{O}$ SYSTEM MAGAZINE returns to the pump 
after going through the filter process. The storage tank provides additional $\mathrm{D}_{2} \mathrm{O}$ that is required. The pump supplies $\mathrm{D}_{2} \mathrm{O}$ to its other parts, the Common Bleed Valve and the Series Valve using pressurization of $\mathrm{D}_{2} \mathrm{O}$.

\subsection{Control System}

The Common Bleed Valve pressure is controlled by the PC11 using the measured values from the PT11. The dualization mode has been set during the mode change to ensure the pressure does not exceed the limit of the safety relief valve when reaching the peak pressure of transient state. In order to maintain a constant Common Bleed Valve pressure as a set value, $\mathrm{PC} 11$ computes the control signal of PCV11 using the set control value and feedback signal.

PCV11\#1(A), a Serial Valve is set for each selected operation mode and slew rate is applied to prevent control instability due to a sudden opening or closing of the respective valve. The closed value of the series valve for each operating mode is input or modified by the operator by pressing the buttons on the controller front panel.

PCV11\#2(A), a Load Shunt Valve calculates the drive control signal through the PI control loop with the control setpoint and feedback input to maintain the drive pressure of $\mathrm{F} / \mathrm{M}$ according to the set value. In the case of high-pressure mode operation, the conditions of the process system frequently change during the fuel change process, and pressure fluctuations continue due to those frequent changes, which may cause excessive wear in the valve if they continue for a long time. The proposed control scheme is designed to overcome such problems, and it is described in Section 4.

The $\mathrm{F} / \mathrm{H}$ system does not have a spare controller, so if any of the three controllers fails during the fuel change, the heavy water supply pressure control will become impossible. This can result in heavy water leaks and a difficult recovery of the heavy water supply system due to uncontrollable conditions. That is, the system characteristics such as valve parameters, flow rate, and pressure are not properly reflected in the control logic, and excessive pressure occurs during the operation mode switching. A simulator based on actual operation data was developed for this study and in this case the proposed control method was tested in order to check if the proposed $\mathrm{F} / \mathrm{H}$ system is stable even if it is affected by the valve nonlinear characteristics, the process time delay, and valve nonlinear characteristics.

\section{Development of a Simulator}

\subsection{F/H System Block Diagram}

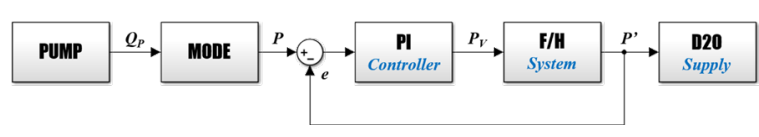

Figure 2. Control block diagram

Figure 2 shows a diagram of the control block typical of the $\mathrm{F} / \mathrm{H}$ system. The pump operates in any operation mode and maintains a constant flow rate such as $Q_{P}=72 G P M$. The set values of the Common Bleed Valve(PC) and $\mathrm{D}_{2} \mathrm{O}$ supply pressure (PLA, A-side pressure level; PLC, $\mathrm{C}$-side pressure level) are chosen according to the system mode.

The $\mathrm{F} / \mathrm{H}$ system uses the valve opening $\left(\mathrm{P}_{\mathrm{V}}\right)$, which is the output of the PI controller, to determine the flow coefficient $\left(C_{V}\right)$. Next, one should determine the equivalent flow coefficient, which is used for calculating the equivalent flow rate ( $Q$ ). Finally, the Common Bleed Valve pressure (PC) and the heavy water supply pressure (PLA, PLC) are calculated using $Q$. The flow coefficient determines the volume flowing through the pipeline and this volume is used to determine the value of the pressure that should be supplied to be supplied to the heavy water.

Therefore, it is very important to determine the relationship between flow coefficients and each connected valve in order to develop an exact simulator. The relationship between $\mathrm{P}_{\mathrm{v}}$ and $\mathrm{C}_{\mathrm{v}}$ can be obtained based on the characteristic curve of the valve (Control Component Inc., USA). The valve constant is expressed as a function which consists of the flow $\mathrm{Q}$ and the pressure difference between the valves $(\triangle \mathrm{P})$ as follows.

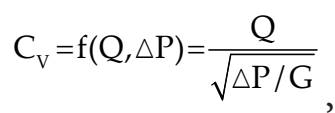

Here, G represents the specific weight of fluid and depends on the temperature. The specific 
weight of fluid, that is $1.1[\mathrm{~g} / \mathrm{ml}]$, was selected under steady-state operating conditions. $\mathrm{Q}$ and $\triangle \mathrm{P}$ are derived from Eq. (1), which is the orifice equation.

$$
\begin{aligned}
& Q=C_{V} \times \sqrt{\Delta P / G}, \\
& \Delta P=G \frac{Q^{2}}{\left(C_{V}\right)^{2}},
\end{aligned}
$$

\subsection{Equivalent Valve Coefficient}

Figure 3 shows a diagram that contains flow coefficient needed for calculating the system modelling. The equivalent equation expressing the series-parallel flow coefficient is derived below. In the case of connecting $\mathrm{C}_{\mathrm{V} 1}$ and $\mathrm{C}_{\mathrm{V} 2}$ in series, $\mathrm{C}_{\mathrm{VT}}$ can be derived from $\mathrm{P}_{\mathrm{T}}=\mathrm{P}_{1}+\mathrm{P}_{2}$ and Eq. (3). To summarize, $P_{T}$ is represented as:

$$
P_{T}=G \frac{Q_{T}^{2}}{\left(C_{V T}\right)^{2}}=G \frac{Q_{1}^{2}}{\left(C_{V 1}\right)^{2}}+G \frac{Q_{2}^{2}}{\left(C_{V 2}\right)^{2}},
$$

The flow rates of the series connections of the orifice valve are the same, thus, $Q_{T}^{2}=Q_{1}^{2}=Q_{2}^{2}$. Therefore, $\frac{1}{\left(\mathrm{C}_{\mathrm{VT}}\right)^{2}}=\frac{1}{\left(\mathrm{C}_{\mathrm{V} 1}\right)^{2}}+\frac{1}{\left(\mathrm{C}_{\mathrm{V} 2}\right)^{2}}$ and can be summarized as shown in $\mathrm{Eq}(5)$.

$$
C_{V T}=\frac{1}{\sqrt{\frac{1}{\left(C_{V 1}\right)^{2}}+\frac{1}{\left(C_{V 2}\right)^{2}}}},
$$

The equivalent flow coefficient for two valves which are connected in parallel $\mathrm{Q}_{\mathrm{T}}=\mathrm{Q}_{1}+\mathrm{Q}_{2}$ from Eq. (2) is satisfied as follows.

$$
Q_{T}=C_{V T} \sqrt{\triangle P / G}=C_{V 1} \sqrt{\triangle P / G}+C_{V 2} \sqrt{\triangle P / G}
$$

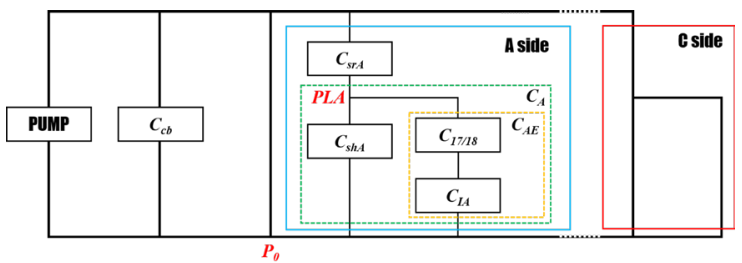

Figure 3. $\mathrm{D}_{2} \mathrm{O}$ supply block diagram as valve coefficient

The pipeline pressure, which is exerted by the return pump, is constant at $\mathrm{P}_{0}=50 \mathrm{psi}$. Therefore, the pressure difference between both $\mathrm{C}_{\text {shA }}$ and $\mathrm{C}_{\mathrm{AE}}$ is $\triangle \mathrm{P}=\mathrm{PLA}-\mathrm{P}_{0}$. On the other hand, because the parallel connections of the orifice valve and differential pressure are the same, $\sqrt{\triangle P / G}$ can be omitted, as it is shown in Eq. (7).

$$
C_{V T}=C_{V 1}+C_{V 2}
$$

The valve coefficient of the heavy water supply system can be illustrated as follows. First, in Figure 3, the equivalent flow coefficient $\mathrm{C}_{\mathrm{AE}}$ is connects $C_{17 / 18}$ (which is the A-side load) to $C_{I A}$ in series. Using Eq. (5), it can be expressed as:

$$
C_{A E}=\frac{1}{\sqrt{\frac{1}{C_{17 / 18}^{2}}+\frac{1}{C_{I A}^{2}}}}
$$

Next, $C_{A}$ is an equivalent valve coefficient and connects $\mathrm{C}_{\mathrm{AE}}$ to $\mathrm{C}_{\mathrm{shA}}$ in parallel. Using Eq. (7), $\mathrm{C}_{\mathrm{A}}$ can be expressed as follows.

$C_{A}=C_{A E}+C_{s h A}$

Finally, $\mathrm{CT}_{\mathrm{A}}$ is an A-side equivalent valve coefficient as it is expressed by Eq. (8).

$$
C T_{A}=\frac{1}{\sqrt{\frac{1}{C_{A}^{2}}+\frac{1}{C_{s r A}^{2}}}}
$$

The C-side as the supply of heavy water is calculated in the same way as that for the A-side of the heavy water supply pressure. To sum up, the total equivalent valve coefficient of the supply of heavy water, CT , is shown in Eq. (11). Here, $C_{c b}$ represents the coefficient of Common Bleed Valve.

$$
C T=C_{c b}+C T_{A}+C T_{C}
$$

\subsection{Equivalent Flow \& Heavy Water Pressure Supply System}

Series Valve flow is summarized using Eq. (2).

$$
Q_{s r A}=C_{s r A} \sqrt{P C-P L A}
$$

This is similarly applied to the C-side,

$$
Q_{s r C}=C_{s r C} \sqrt{P C-P L C}
$$

The Common Bleed Valve flow can be calculated from Eq. (14) using Eq. (12) \& (13). 


$$
Q_{c b}=Q_{P}-Q_{s r A}-Q_{s r C}
$$

Here, Quantity of pump $\left(\mathrm{Q}_{\mathrm{p}}\right)$ is located immediately downstream of the pump and is the volume flowing on top of the Common Bleed Valve and Series Valve. Calculations using the previously obtained $\mathrm{CT}, \mathrm{C}_{\mathrm{A}}, \mathrm{C}_{\mathrm{C}}, \mathrm{Q}_{\mathrm{srA}}$ and $\mathrm{Q}_{\mathrm{srC}}$ in Eq. (3) can be expressed as follows.

$$
P C=G\left(\frac{Q_{P}}{C T}\right)^{2}, P L A=G\left(\frac{Q_{s r A}}{C_{A}}\right)^{2} \quad P L C=G\left(\frac{Q_{s r C}}{C_{C}}\right)^{2}
$$

Here, $\mathrm{PC}$ is the pressure of the Common Bleed Valve, and PLA and PLC are the $\mathrm{A}$-side and $\mathrm{C}$-side pressures of the heavy water, respectively.

\subsection{Wolsong Reactor Simulation}

A simulated experiment was carried out under the same conditions as those of the actual process of data generation for the Wolsong reactor. For the simulated experiment, the following conditions were applied: a sampling time of $0.05 \mathrm{sec}$, a mode change of the type low-medium-high, and the PI controller was applied to the PC, PLA, and PLC.

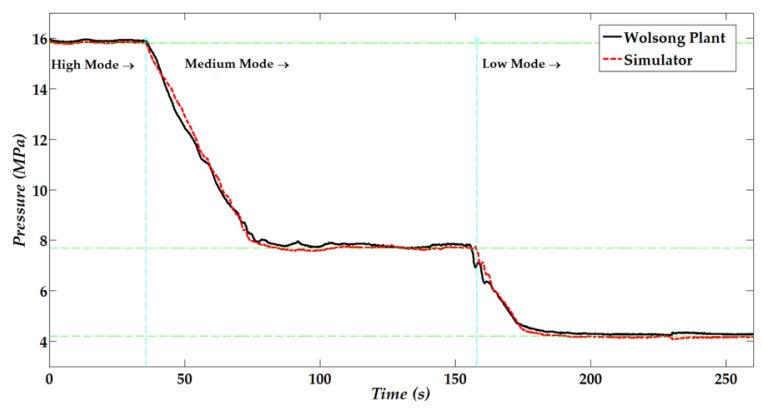

Figure 4. Comparison of the response of the Wolsong Plant and of the simulator (High Mode: F/M work for fuel exchange, Medium Mode: Reactor vault work, Low Mode: New fuel loading, spent fuel emission)

The Wolsong reactor chamber process data and the values of the simulator are shown in Figure 4 . The solid line represents the real data peculiar to the Wolsong nuclear power plant and the dotted line represents the simulation result. The steady-state value of PLA is fairly similar to the output of the Wolsong reactor. The next section introduces a controller for improving the performance of $\mathrm{F} / \mathrm{H}$ using the previously modelled simulator.

\section{Controller Design}

\subsection{Existing Control Methods}

The $\mathrm{F} / \mathrm{H}$ system is operated by PI controllers mounted at Common Bleed Valves and Load Shunt Valves and the overall control function can be expressed as follows.

$$
C O(s)=K_{S}\left(1+\frac{1}{T R \cdot S}\right) \frac{100}{P B} D V(s)+F F(s)
$$

Here, $\quad K_{S}=\frac{\text { Spanof the controller output }}{\text { Spanof the processinput }}=5$ and is a scaling factor. From equation (16), it can be noticed that, as PB increases, the proportional gain decreases, and as TR increases, the impact of the integral term on the output decreases. Optimal values of $\mathrm{PB}, \mathrm{TR}, \& \mathrm{FF}$ are in Table 1 .

Table 1. Parameters of controller equation

\begin{tabular}{|c|l|c|c|}
\hline Parameter & Description & $\begin{array}{c}\text { Common } \\
\text { Bleed }\end{array}$ & $\begin{array}{c}\text { Load } \\
\text { Shunt }\end{array}$ \\
\hline $\begin{array}{c}\text { PB } \\
\text { Proportional Band }\end{array}$ & $\frac{100}{P B}=K_{P}$ & 0.6667 & 0.8333 \\
\hline $\begin{array}{c}\text { TR(min) } \\
\text { Reset Time }\end{array}$ & $\frac{1}{T R}=K_{I}$ & 0.2222 & 0.2222 \\
\hline $\begin{array}{c}\text { FF(\%) } \\
\text { Feed Forward }\end{array}$ & Bias & $(T R=4.5)$ \\
\hline
\end{tabular}

The $D V(s)$ is the deviation of the measured value from the desired value of the plant output, and $\mathrm{FF}(\mathrm{s})$ is a bias value for initial setting of the valve opening, which contributes to the stabilization of the initial start-up of the process.

\subsection{PINN Control Method}

$\mathrm{F} / \mathrm{H}$ system can cause instability in the process system if high frequency disturbances are included among the process variables. Therefore, the PI controller shall be used without $\mathrm{D}$ as the derivative term. However, in order to be active, the PI controller must respond to changes in the parameters of the system to compensate for the $\mathrm{F} / \mathrm{H}$ pressure control since they display characteristics of nonlinearity and the time-delay method is used. In this study, the neural network algorithm is connected with the existing PI controller in parallel to compensate for the error caused by changes in the parameters 
involved. The controller was designed in order to reduce the excessive oscillations that may occur when the mode of heavy water supply pressure changes.

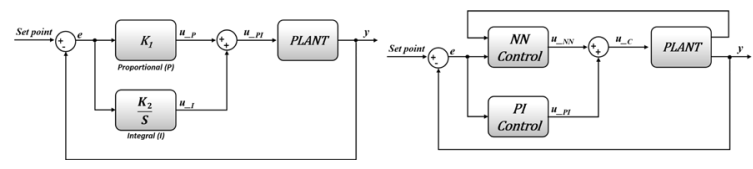

Figure 5. Controller structure (Left: PI, Right: PINN)

Figure 5 shows the structure of the PINN controller. The control output, $\mathrm{U}_{\mathrm{PI}}$, can be calculated using the error from the difference between the measured value and the desired value of the plant output according to the operation mode. The neural network computes the control output $\mathrm{U}_{\mathrm{NN}}$ using the error that affects the system, the set pressure, the plant output, and valve pressures as learning data. Finally, the control output of PINN is derived from outputs from PI control and NN control, and the function can be written as $\mathrm{U}_{\mathrm{C}}=\mathrm{U}_{\mathrm{PI}}+\mathrm{U}_{\mathrm{NN}}$. The plant output, $\mathrm{y}$, is $\mathrm{D}_{2} \mathrm{O}$ supply pressure. A feed-forward neural network structure was applied to PINN.

The structure is composed of three layers namely the input, hidden, and output layer, in order to reduce its complexity but without reducing its performance. The input layer uses eight learning inputs, including the bias, the hidden layer consists of 24 units, and the output layer has one output to compensate for the A-side of the heavy water supply pressure. The data used in the input layer are $\mathrm{e}(\mathrm{k}), \mathrm{e}(\mathrm{k}-1)$ : current and previous error value, PLA_S: set value of A-side, PLA_P: measured value of A-side, PC_S: set value of Common Bleed Valve, PC_P: measured value of Common Bleed Valve and SRA: measured value of Series Valve.

\section{Simulation}

It is important that the $\mathrm{F} / \mathrm{H}$ system operate within the set safety point for a stable system behaviour as well for reducing the occurrence of oscillation and error when there is a change in the environment or operation mode. This section discusses the simulated operation of applying changing parameters to the $\mathrm{F} / \mathrm{H}$ model with PINN controller. These changing parameters are the Series Valve's slew rate change and the modified valve characteristics of the shunt valve. On the other hand, the pressure operation mode scenario was set to Low $\rightarrow$ High $\rightarrow$ Medium $\rightarrow$ Low mode.

\subsection{Slew Rate Change of Series Valve}

The Series Valve uses its slew rate without a separate controller when reducing the rapid pressure changes during the operation of nuclear reactors. The slew rate utilizes both automatic and manually set settings to determine if the valve is to be opened. The system may be unstable if the operator sets the wrong values based on the user's manual. Therefore, simulations were done to verify if the Series Valve works properly in accordance with changes in the slew rate when the PINN controller is utilized. Figures 6(a) 6(d) show the results when the initial SR values, increasing slew rate $\alpha=0.5810$ and decreasing slew rate $\beta=-0.5380$, are changed.

Decreasing $\alpha$ from 0.5810 to 0.4310 yields the result shown in Figure 6(a). Both PI and PINN controllers exhibit similar characteristics with Low $\rightarrow$ High pressure mode change. PINN reached the reference input faster than PI by 5.4 seconds but there was undershooting in the case of the High $\rightarrow$ Medium mode change. On the other hand, the PINN controller reached the set value faster than the PI by 4.0 seconds and without undershooting for the Medium $\rightarrow$ Low mode change. The difference in root mean square error (RMSE) between the PINN and PI controllers is 0.1053 .

Figure $6(\mathrm{~b})$ shows the result when $\beta$ is increased from -0.5810 to -0.3880 . PINN reached the reference value 1.8 seconds faster than PI without overshooting when for the Low $\rightarrow$ High mode change. Both controllers undershot in case of the High $\rightarrow$ Medium mode change. The PINN controller's RMSE for the entire interval is better than PI's by 0.0883 .

The result when decreasing $\beta$ from -0.5810 to -0.6880 is given in Figure 6(c). PINN reached the set value faster than PI by 4.4 seconds for the High $\rightarrow$ Medium mode change while the PINN RMSE was better than PI RMSE by 0.0411 for the whole interval. Increasing $\alpha$ from 0.5810 to 0.7310 gave the result shown in Figure 6(d). PINN controller reached the set value 3.0 seconds faster 


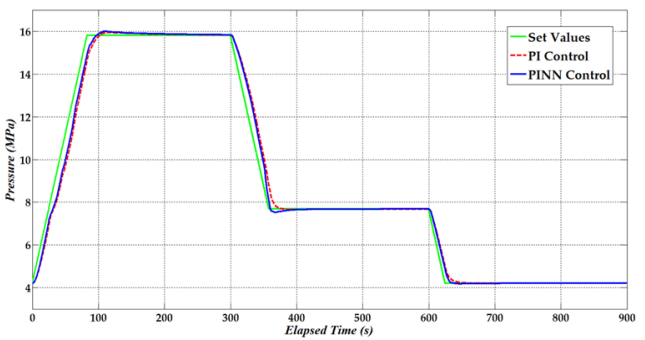

(a) PLA pressure plot for $\alpha=0.4310, \beta=-0.5380$

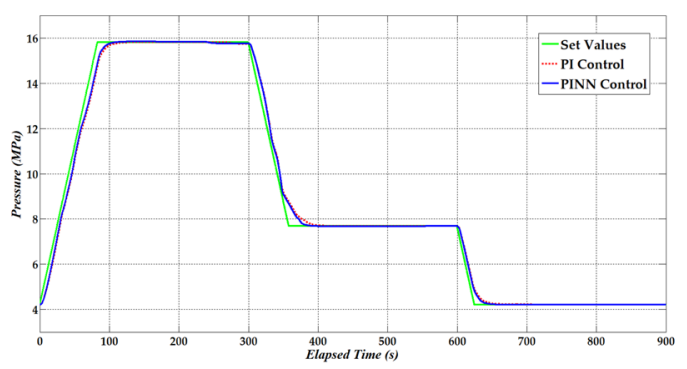

(c) PLA pressure plot for $\alpha=0.5810, \beta=-0.6880$

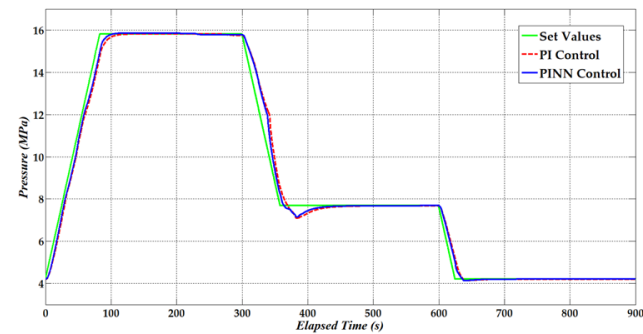

(b) PLA pressure plot for $\alpha=0.5810, \beta=-0.3880$

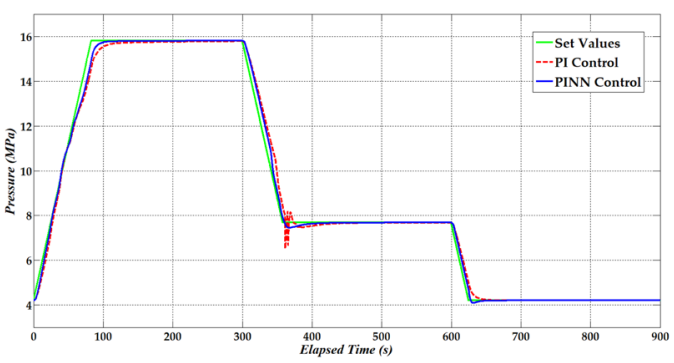

(d) PLA pressure plot for $\alpha=0.7310, \beta=-0.5380$

Figure 6. Performance comparison between the PINN and PI controllers for the set values NOTE: $\alpha$ and $\beta$ indicate increasing slew rate and decreasing slew rate of Series Valves, respectively

than PI without overshooting when for the Low $\rightarrow$ Medium mode change. The oscillation was reduced by the PINN for the High $\rightarrow$ Medium mode change while for the PI it was not possible.

The PINN controller's performance is analyzed when the time-domain specification included a change in process mode at the rate of 300 seconds per section. The set value was reached faster and more accurately by PINN as compared to PI especially when a Low $\rightarrow$ High mode change as it can be seen in Figure 6(d). It can also be noticed that the $\mathrm{F} / \mathrm{H}$ system was not properly controlled for 150 seconds by the PI controller as it can be proved by the presence of oscillation which was otherwise reduced by the PINN controller. The simulation results showed that PINN control method displays a lower error than the PI control method. The PINN stabilizes the system by reducing oscillation while oscillation still occurs in some cases when the PI controller is utilized. Table 2 summarizes the control performance.

\subsection{Load Shunt Valve Curve}

The characteristic curve of the Load Shunt Valve is used as a specification, which includes percent of rated travel vs. valve flow coefficient, and it can be used in calculating the volume of flow. Figure 8 to 11 show the result of simulations that were carried out based on the changing characteristic curve with the assumption that its value would change due to wear and corrosion. The solid and dotted lines in Figures 7(a) to 7(d) represent the characteristic curve of the Load Shunt Valve before and after the above-mentioned changes, respectively. Figures 8 to 11 show plot of PINN and PI controllers with respect to heavy water supply pressure when various characteristic curves of Load Shunt Valves are applied.

The curve in Figure 7(a) is approximately 3.4 times as steep as the original curve for the $0 \%$ $24 \%$ interval and it has a Dead-band to check the interactive effect of valve controllers for the $25 \%$ $-64 \%$ interval.

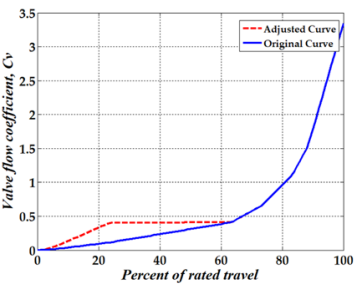

(a) Case study 1

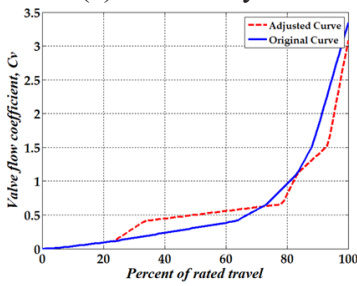

(c) Case study 3

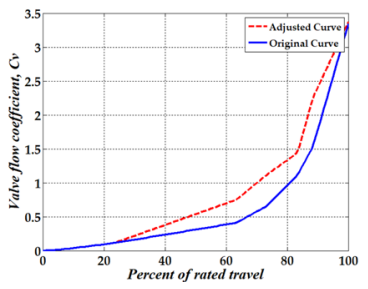

(b) Case study 2

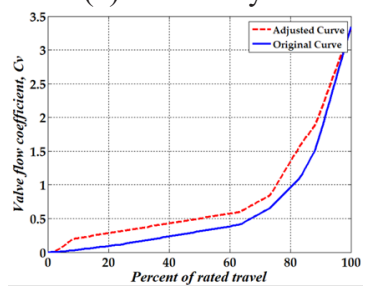

(d) Case study 4
Figure 7. Load Shunt valve characteristic curve 
Figure 8 shows the supply pressure waveform of the heavy water when the characteristic curve of the Load Shunt Valve changes from $0 \%$ to $64 \%$. The PINN controller reaches the set value 9.8 seconds quicker than the PI for the Low $\rightarrow$ High mode change. The PI cannot properly deal with oscillations during the High $\rightarrow$ Low mode change while PINN can compensate for it. The overall PINN RMSE is 0.1076 lower than PI.

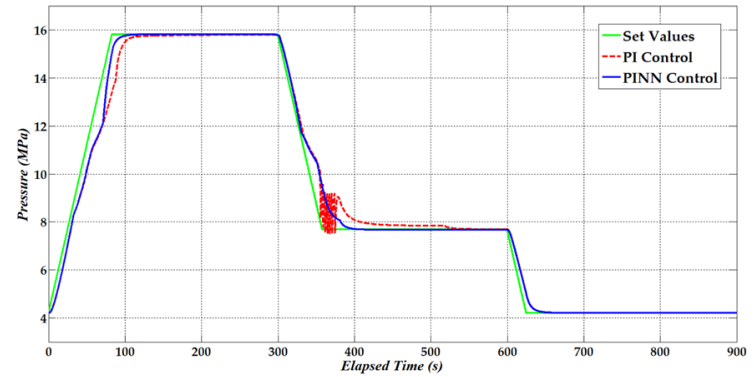

Figure 8. PLA pressure waveform curve for Fig. 7(a)

The adjusted characteristic curve in Figure 7(b) has a bigger flow coefficient than the original curve for the given interval. Figure 9 shows the supply pressure waveform of the heavy water when the characteristic curve of the Load Shunt Valve changes from $24 \%$ to $100 \%$. The PINN controller was faster than PI in reaching the set values by 1 second and 7.2 seconds for the High $\rightarrow$ Medium and Medium $\rightarrow$ Low mode changes, respectively. The overall PINN RMSE for this case was lower than the PI controller by 0.0845 .

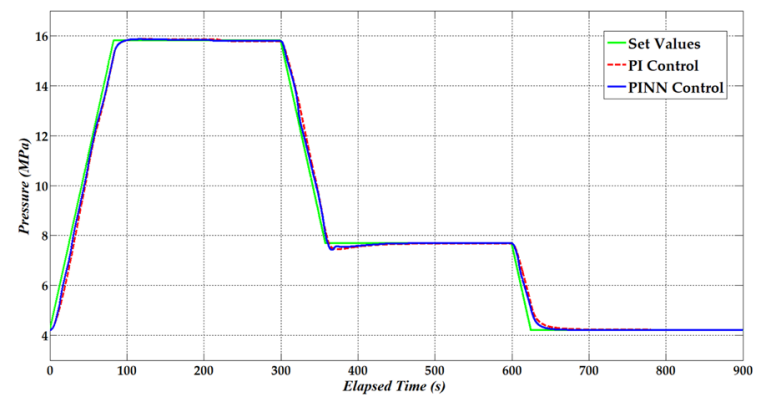

Figure 9. PLA pressure waveform for Fig. 7(b)

Figure 10 shows the supply pressure waveform of the heavy water when the characteristic curve of the Load Shunt Valve changes from 24\% to $100 \%$. The intricately modified characteristic curve in Figure 7(c) was applied. The PI controller reached the set value 2.6 seconds faster than the PINN for the Low $\rightarrow$ High mode change. On the other hand, PINN reached the set values faster than PI by 8 seconds and by 71.4 seconds for the High $\rightarrow$ Medium and Medium $\rightarrow$ Low mode changes, respectively. The overall PINN controller RMSE for this case was lower than that of the PI controller by 0.2939 .

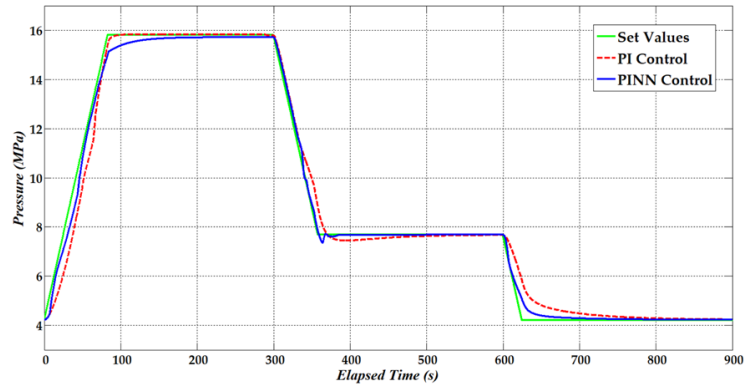

Figure 10. PLA pressure waveform for Fig. 7(c)

Figure 11 shows the supply pressure waveform of the heavy water when the characteristic curve of the Load Shunt Valve changes for the whole interval as it is illustrated in Figure 7(d). The PINN controller was able to reach the set values faster than PI controller by 2.8 seconds, 3.5 seconds, and 19.6 seconds for the Low $\rightarrow$ High, High $\rightarrow$ Medium and Medium $\rightarrow$ Low mode changed, respectively. The overall PINN controller RMSE was higher than that of the PI controller by 0.1113 . Overall, Figures 8 to 11 show that PINN controller responds more accurately and more rapidly than PI controller.

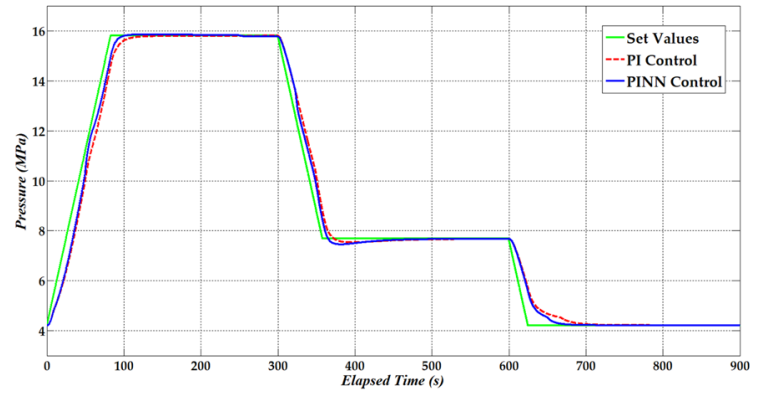

Figure 11. PLA pressure waveform for Fig. 7(d)

Simulations showed that PINN control had a lower error than the PI control. The PINN stabilized the system by reducing oscillation while oscillation still occurred in some cases when the PI controller was utilized.

To sum up, the simulation results as it is shown in Table 2, the PINN control method was able to accurately control valves with a lower RMSE error than the PI control method for all the case studies involved. In addition, oscillation, which is an important factor in the nuclear power plant, occurred in two cases when using 
the PI controller while this did not happen for the PINN. The PINN control method reduces RMSE error and does not cause oscillation which helps perform a stable operation and use the heavy water efficiently.

Table 2. Controller performance for various cases

\begin{tabular}{|c|c|c|c|}
\hline \multicolumn{4}{|c|}{ Varying series valve slew rate } \\
\hline Case & Controller & RMSE & Oscillation \\
\hline$\alpha=0.4310$ & PI & 0.5138 & - \\
\hline$\beta=-0.5380$ & PINN & 0.4130 & - \\
\hline$\alpha=0.4310$ & PI & 0.4440 & - \\
\hline$\beta=-0.5380$ & PINN & 0.3557 & - \\
\hline$\alpha=0.4310$ & PI & 0.3362 & - \\
\hline$\beta=-0.5380$ & PINN & 0.2951 & - \\
\hline$\alpha=0.4310$ & PI & 0.3539 & $\mathrm{O}$ \\
\hline$\beta=-0.5380$ & PINN & 0.2319 & - \\
\hline \multicolumn{4}{|c|}{ Varying load shunt valve characteristics } \\
\hline Case & Controller & RMSE & Oscillation \\
\hline Dead band & PI & 0.5742 & $\mathrm{O}$ \\
\hline$(0 \sim 64 \%)$ & PINN & 0.4660 & - \\
\hline Higher COEF & PI & 0.3285 & - \\
\hline$(24 \sim 100 \%)$ & PINN & 0.2440 & - \\
\hline Mixed COEF & PI & 0.5465 & - \\
\hline$(24 \sim 100 \%)$ & PINN & 0.2526 & - \\
\hline Higher COEF & PI & 0.5285 & - \\
\hline$(0 \sim 100 \%)$ & PINN & 0.4172 & - \\
\hline
\end{tabular}

\section{Conclusion}

A system similar to the $\mathrm{F} / \mathrm{H}$ system for the Wolsong nuclear power plants was designed and simulated

\section{REFERENCES}

Attaran, S. M., Yusof, R. \& Selamat, H. (2016). A novel optimization algorithm based on epsilon constraint-RBF neural network for tuning PID controller in decoupled HVAC system, Applied Thermal Engineering, 99, 613-624.

Azali, S. \& Sheikhan, M. (2016). Intelligent control of photovoltaic system using BPSOGSA-optimized neural network and fuzzy-based PID for maximum power point tracking, Applied Intelligence, 44(1), 88-110.

Banerjee, S. \& Gupta, H. P. (2017). The evolution of the Indian nuclear power programme, Progress in Nuclear Energy, 101, 4-18. in this paper. The system performance was tested by checking its output for various modes of the actual process. The proposed PINN controller was used to reduce the process instability that occurred when there was a change which affected the parameters involved. The proposed system reflected both the process and control system characteristics of the $\mathrm{F} / \mathrm{H}$ system and it also confirmed that the process characteristics correctly simulated the actual process data.

The proposed PINN controller has the PI and neural network controllers that are connected in parallel to withstand parameter changes. The characteristics of the Load Shunt Valve and the Series Valve's slew rate were adjusted during simulations in order to analyze the controller's performance. The use of the PI controller involved some oscillation and displayed a higher RMSE while the PINN controller reduced the oscillation and reached the set values more quickly. Additionally, the Common Bleed Valve did not exceed the set safety pressure in the operation mode.

The PINN controller can help to adaptively improve the performance of the $\mathrm{F} / \mathrm{H}$ system when the involved parameters change and disturbances occur.

\section{Acknowledgements}

This research was supported by the Brain Research Program of the National Research Foundation (NRF) funded by the Korean government (MSIT) (No. NRF-2017M3C7A 1044816).

Beschi, M., Padula, F. \& Visioli, A. (2016). Fractional robust PID control of a solar furnace, Control Engineering Practice, 56, 190-199.

Borkowski, D. (2017). Maximum Efficiency Point Tracking (MEPT) for Variable Speed Small Hydropower Plant With Neural Network Based Estimation of Turbine Discharge, IEEE Transactions on Energy Conversion, 32(3), 1090-1098.

Griffiths, M. (2013). The Effect of Irradiation on $\mathrm{Ni}$-containing Components in CANDU ${ }^{\circledR}$ Reactor Cores: A Review, AECL Nuclear Review, 2(1), 1-16. DOI: 10.12943/ANR.2013.00001 
Heravi, G. \& Eslamdoost, E. (2015). Applying Artificial Neural Networks for Measuring and Predicting Construction-Labor Productivity, Journal of Construction Engineering and Management, 141(10), 04015032-1-11.

Kadiyala, A., Kommalapati, R. \& Huque, Z. (2016). Quantification of the Lifecycle Greenhouse Gas Emissions from Nuclear Power Generation Systems, Energies, 9(11), 863-876.

Katoh, A., Chikazawa, Y., Obata, H. \& Kotake, S. (2010). Development of Advanced Fuel Handling Machine for JSFR, Journal of Nuclear Science and Technology, 47(7), 642-651.

Lamba, R., Singla, S. K. \& Sondhi, S. (2017). Fractional order PID controller for power control in perturbed pressurized heavy water reactor, Nuclear Engineering and Design, 323, 84-94.

Li, S., Wang, P. \& Goel, L. (2015). A Novel Wavelet-Based Ensemble Method for Short-Term Load Forecasting with Hybrid Neural Networks and Feature Selection, IEEE Transactions on Power Systems, 31(3), 1788-1798.

Liu, J., Fang, W., Zhang, X. \& Yang, C. (2015). An Improved Photovoltaic Power Forecasting Model With the Assistance of Aerosol Index Data, IEEE Transactions on Sustainable Energy, 6(2), 434-442.

Liu, X., Zhang, Y. \& Lee, K. Y. (2016). Coordinated distributed MPC for load frequency control of power system with wind farms, IEEE Transactions on Industrial Electronics, 64(6), 5140-5150.

Mahajan, S., Liu, H. M., Tsai, T. C. \& Chen, L. J. (2018). Improving the Accuracy and Efficiency of PM2.5 Forecast Service Using Cluster-Based Hybrid Neural Network Model, IEEE Access, 6, 19193-19204.

Nikolovski, S., Reza Baghaee, H. \& Mlakić, D. (2018). ANFIS-Based Peak Power Shaving/ Curtailment in Microgrids Including PV Units and BESSs, Energies, 11(11), 2953-2976.

Prasad, G., Swidenbank, E. \& Hogg, B. W. (1998). A Local Model Networks Based
Multivariable Long-Range Predictive Control Strategy for Thermal Power Plants, Automatica, 34(10), 1185-1204.

Qin, Y., Sun, L., Hua, Q. \& Liu, P. (2018). A Fuzzy Adaptive PID Controller Design for Fuel Cell Power Plant, Sustainability, 10(7), 2438.

Rahman,A., Saikia, L. C., \& Sinha, N. (2016).AGC of dish-Stirling solar thermal integrated thermal system with biogeography based optimised three degree of freedom PID controller, IET Renewable Power Generation, 10(8), 1161-1170.

Shah, U. H., Hong, K. \& Choi, S. (2017). OpenLoop Vibration Control of an Underwater System: Application to Refueling Machine, IEEE/ASME Transactions on Mechatronics, 22(4), 1622-1632.

Sharma, R., Kumar, V., Gaur, P. \& Mittal, A. P. (2016). An adaptive PID like controller using mix locally recurrent neural network for robotic manipulator with variable payload, ISA transactions, 62, 258-267.

Vazquez, S., Rodriguez, J., Rivera, M., Franquelo, L. G. \& Norambuena, M. (2017). Model Predictive Control for Power Converters and Drives: Advances and Trends, IEEE Transactions on Industrial Electronics, 64(2), 935-947.

Zarei, M. (2018). A multi-point kinetics based MIMO-PI control of power in PWR reactors, Nuclear Engineering and Design, 328, 283-291.

Zeng, D., Zheng, Y., Luo, W., Hu, Y., Cui, Q., Li, Q. \& Peng, C. (2019). Research on Improved Auto-Tuning of a PID Controller Based on Phase Angle Margin, Energies, 12(9), 1704-1720.

Zeng, M., Song, Y., Bai, Y., Wang, M., Wang, J. \& Yu, J. (2018). Simulation and Test of Fuel Handling System for Accelerator Driven Subcritical Reactor, Annals of Nuclear Energy, $116,388-394$.

Zhang, J. \& Shen, T. (2016). Real-Time Fuel Economy Optimization With Nonlinear MPC for PHEVs, IEEE Transactions on Control Systems Technology, 24(6), 2167-2175. 